\title{
Neuropilins are positive regulators of Hedgehog signal transduction
}

\author{
R. Tyler Hillman, ${ }^{1,7}$ Brian Y. Feng, ${ }^{2}$ Jun $\mathrm{Ni}^{2}{ }^{2}$ Wei-Meng Woo, ${ }^{3}$ Ljiljana Milenkovic, ${ }^{1,4,5,7}$ \\ Melanie G. Hayden Gephart, ${ }^{6,7}$ Mary N. Teruel, ${ }^{2}$ Anthony E. Oro, ${ }^{3}$ James K. Chen, ${ }^{2}$ \\ and Matthew P. Scott ${ }^{1,4,5,7,8}$ \\ ${ }^{1}$ Department of Genetics; ${ }^{2}$ Department of Chemical and Systems Biology; ${ }^{3}$ Program in Epithelial Biology; ${ }^{4}$ Department \\ of Bioengineering; ${ }^{5}$ Department of Developmental Biology; ${ }^{6}$ Department of Neurosurgery, ${ }^{7}$ Howard Hughes Medical Institute, \\ Stanford University School of Medicine, Stanford, California 94305, USA
}

\begin{abstract}
The Hedgehog $(\mathrm{Hh})$ pathway is essential for vertebrate embryogenesis, and excessive Hh target gene activation can cause cancer in humans. Here we show that Neuropilin 1 (Nrp1) and Nrp2, transmembrane proteins with roles in axon guidance and vascular endothelial growth factor (VEGF) signaling, are important positive regulators of Hh signal transduction. Nrps are expressed at times and locations of active Hh signal transduction during mouse development. Using cell lines lacking key Hh pathway components, we show that Nrps mediate Hh transduction between activated Smoothened (Smo) protein and the negative regulator Suppressor of Fused (SuFu). Nrp1 transcription is induced by $\mathrm{Hh}$ signaling, and Nrp1 overexpression increases maximal Hh target gene activation, indicating the existence of a positive feedback circuit. The regulation of Hh signal transduction by Nrps is conserved between mammals and bony fish, as we show that morpholinos targeting the Nrp zebrafish ortholog nrp1a produce a specific and highly penetrant $\mathrm{Hh}$ pathway loss-of-function phenotype. These findings enhance our knowledge of Hh pathway regulation and provide evidence for a conserved nexus between Nrps and this important developmental signaling system.
\end{abstract}

[Keywords: neuropilins; Hedgehog pathway; development; zebrafish; primary cilium]

Supplemental material is available for this article.

Received June 25, 2011; revised version accepted September 29, 2011.

The Hedgehog $(\mathrm{Hh})$ signaling pathway is a conserved mode of cell-cell communication. Hh signaling is essential for mammalian cell fate specification, cell proliferation, and epithelial-mesenchymal interactions (Ingham and McMahon 2001; Beachy et al. 2004). Misregulated signaling is a cause of human cancers such as basal cell carcinoma (BCC) and medulloblastoma (MB) (Beachy et al. 2004). Patched1 (Ptc1) is a 12-pass transmembrane protein that inhibits Hh pathway activation by blocking the action of Smoothened (Smo), a seven-pass transmembrane protein. Hh ligands (Sonic hedgehog [Shh], Desert hedgehog, or Indian hedgehog [Ihh]) bind to Ptc1 and promote Smo activation (Corbit et al. 2005; Rohatgi et al. 2007; Goetz and Anderson 2010). Smo then acts through an unknown mechanism to inhibit the cytoplasmic protein Suppressor of Fused (SuFu), which in turn is a negative regulator of the three mammalian Gli transcription factors (Gli1-3) (Chen et al. 2009; Humke et al. 2010; Wen et al. 2010). Gli3 is

${ }^{8}$ Corresponding author.

E-mail mscott@stanford.edu.

Article published online ahead of print. Article and pubication date are online at http://www.genesdev.org/cgi/doi/10.1101/gad.173054.111. Freely available online through the Genes \& Development Open Access option. predominantly a negative regulator of Hh signaling. In the absence of Hh ligand, full-length Gli3 (Gli3FL) is proteolytically processed into a truncated moiety (Gli3R) that represses Hh target gene expression (Wang et al. 2000). Although Gli2 also exists in two forms and may have repressor activity in some circumstances, the molecule is predominantly an activator of $\mathrm{Hh}$ target gene transcription (Bai and Joyner 2001). Glil is exclusively an activator of $\mathrm{Hh}$ pathway transcription, and Gli1 transcription is strongly induced by Hh signaling as part of a positive feedback loop (Lee et al. 1997). The negative Hh pathway regulator Ptc1 is also a transcriptional target of Hh signaling (Goodrich et al. 1996). Thus, the pathway regulates production of its own components for the purpose of buffering or amplifying the response to ligand (Ingham and McMahon 2001).

In this study, we report discovering the positive actions of neuropilin (Nrp) proteins on Hh signal transduction. Mammals have two Nrp genes, Nrp1 and Nrp2, that encode proteins sharing $\sim 44 \%$ identity at the amino acid level. Nrps have five external domains, a single transmembrane domain, and an 40-amino-acid cytoplasmic domain lacking any recognizable enzymatic structural motifs (Geretti et al. 2008). Nrps contain two tandem extracellular CUB (complement/Uegf/Bmp1) domains, followed 
by a pair of domains with distant homology with clotting factors V/VIII, as well as a membrane-proximal meprin MAM domain. Nrps act in conjunction with transmembrane A-type Plexin proteins as coreceptors for class 3 Semaphorins (Semas), providing a repellent axon guidance signal (Chen et al. 1997; He and Tessier-Lavigne 1997; Kolodkin et al. 1997). Nrp1/PlexinA4 complexes mediate Sema3A signaling, while Sema3F signals through Nrp2/ PlexinA3 complexes (Cheng et al. 2001; Suto et al. 2005; Yaron et al. 2005). Nrp1 has also been identified as a coreceptor for the 165-amino-acid isoform of vascular endothelial growth factor (VEGF165) - a remarkable finding, since Semas share no sequence or structural homology with VEGF proteins (Soker et al. 1998). Nrps serve as coreceptors with the VEGF-R2/Kdr tyrosine kinase receptor, with the VEGF ligand acting as a bridge between the two receptor proteins (Soker et al. 2002; Prahst et al. 2008). Mice mutant for both Nrp genes die at or around embryonic day 8.5 (E8.5) due to severe developmental defects that include errors in yolk sac vasculogenesis, a phenotype that is considerably more severe than that of either single mutant (Kawasaki et al. 1999; Chen et al. 2000; Giger et al. 2000; Takashima et al. 2002).

Here we used an RNAi screen in cultured fibroblasts to identify the multifunctional Nrp molecules as positive regulators of the Hh signaling pathway. We demonstrate that Nrps participate in a positive feedback loop to reinforce $\mathrm{Hh}$ signal transduction and act to regulate the pathway at a level between Smo and SuFu. Inhibition of Nrp functions also blocks $\mathrm{Hh}$ transduction in primary skin cell cultures. We found that the regulation of $\mathrm{Hh}$ signaling by Nrps is evolutionarily conserved, as morpholino (MO) inhibition of zebrafish nrp1a results in a highly penetrant Hh pathway loss-of-function phenotype.

\section{Results}

Identification of Nrps as positive regulators of mammalian Hh signaling

To discover new positive regulators of mouse Hh signal transduction, we conducted an RNAi screen using a library of 816 pools of siRNA that was produced via digestion of long dsRNAs with the enzyme Dicer (diced siRNA pools [DSPs]) (Myers et al. 2003). This library was designed to target regulators of signal transduction, including genes encoding select kinases, phosphatases, and small GTPases, and genes encoding certain recognizable structural elements such as $\mathrm{PH}$ or $\mathrm{SH} 2$ domains (Fig. 1A). The screen was carried out in a special line of NIH3T3 fibroblasts (Shh-LIGHT2 fibroblasts) that were stably transfected with a Glidependent firefly luciferase reporter gene as well as a constitutive Renilla luciferase reporter gene for use in normalization (Taipale et al. 2000). Shh-LIGHT2 fibroblasts were transfected with the diced siRNAs in a 96-well format, then treated for $24 \mathrm{~h}$ with culture medium conditioned with Shh. Hh pathway activity was assessed by measuring the ratio of firefly luciferase activity to Renilla luciferase activity in each well. A minimum threshold for the constitutive Renilla signal was used to filter the results based on cell viability (see the Materials and Methods). After this filter, 691 genes remained (Fig. 1A). All 68 genes that passed a significance threshold $(Z$-score $<-1.5)$ are listed in Supplemental Table S1. Among these genes was Nrp1, which significantly inhibited Shh-induced activation of the Gli-dependent reporter in Shh-LIGHT2 cells (Fig. 1B).

From among our 68 screen hits, we selected Nrp1 for further investigation, for several reasons. Nrp genes are expressed in several locations during development at times when organs and tissues are undergoing Hh-dependent patterning, including in neural tube motor neuron and interneuron precursors (Chen et al. 1997), limb bud mesenchyme (Kitsukawa et al. 1995; Chen et al. 1997), and yolk sac mesoderm (Kawasaki et al. 1999). Nrps are essential for the proper guidance of spinal neuron commissural axons (Zou et al. 2000), a cell population sensitive to Shh attractive cues emanating from the floorplate (Charron et al. 2003). When Kitsukawa and colleagues (Hui and Joyner 1993; Kitsukawa et al. 1995) overexpressed Nrp1 ubiquitously in mice, the majority of surviving animals exhibited preaxial digit duplication reminiscent of that found in Hh pathway gain-of-function mutants.

To confirm the specificity of the Nrp1 RNAi effect, we obtained a synthetic siRNA targeting a region of the Nrp1 coding sequence distinct from that targeted by the original diced pool. As a positive control, we obtained a synthetic siRNA targeting the essential positive Hh pathway regulator Smo. We found that these siRNAs robustly reduced
A

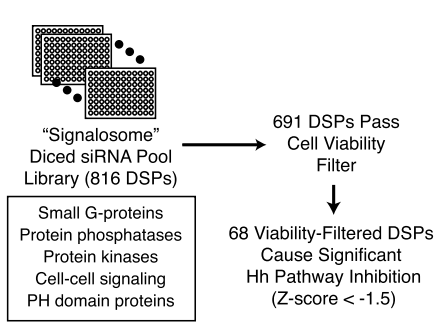

B

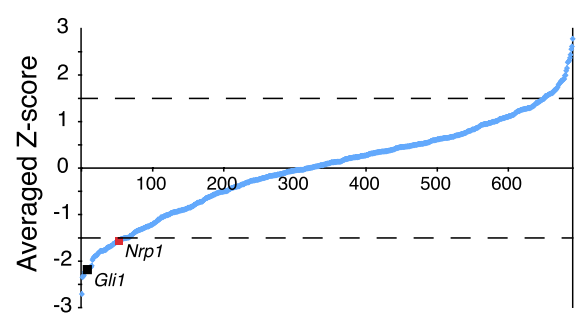

Figure 1. Identification of Nrp1 as a regulator of mammalian $\mathrm{Hh}$ signaling in a cultured cell RNAi screen. (A) A library of DSPs targeting 816 genes implicated in signaling processes was screened using a luciferase-based cell culture assay for $\mathrm{Hh}$ signal transduction. A viability filter was applied to eliminate those DSPs that affected cell survival or growth. Of these viability-filtered DSPs, 68 significantly inhibited Shh-stimulated Gli-dependent luciferase reporter activity (Z-score <-1.5, see Supplemental Table 1). (B) The 691 viability-filtered results from the primary DSP screen are shown in $Z$-score rank order. A DSP targeting Nrp1 (red) significantly blocked Shh-stimulated induction of the Gli-dependent luciferase reporter. A DSP targeting Gli1 also resulted in significant inhibition of Hh signaling (black). 
Nrp1 and Smo protein abundance, respectively, as measured by immunoblot (Supplemental Fig. S1A,B). The synthetic Nrp1 siRNA significantly inhibited Hh luciferase reporter activity in Shh-LIGHT2 fibroblasts, confirming the finding from the initial RNAi screen (Fig. 2A). A synthetic siRNA targeting Nrp2 (Supplemental Fig. 1B) also inhibited Hh pathway activity. When we combined the Nrp1 and Nrp2 siRNAs, the mixture produced enhanced Hh pathway reporter inhibition (Fig. 2A) that was as robust as that caused by Smo RNAi. These data suggest that Nrp1 and Nrp2 have important and partially redundant roles as positive regulators of mammalian $\mathrm{Hh}$ signal transduction. This redundancy caused us to use combined Nrp1+2 RNAi for most subsequent experiments.

As an additional control for off-target effects of Nrp1 RNAi, we tested whether a mouse Nrp1 cDNA could rescue $\mathrm{Hh}$ pathway inhibition caused by siRNA-mediated depletion of endogenous Nrp1. To do this, we treated NIH3T3 fibroblasts with a synthetic siRNA targeting the Nrp1 3' untranslated region (3' UTR). We then transfected these cells with either a Nrp1 expression vector or a vector expressing an unrelated gene (CD4-YFP), along with luciferase reporter plasmids, and stimulated $\mathrm{Hh}$ pathway activity with Shh. We found that Nrp1 cDNA was specifically able to restore the loss of Hh signal transduction caused by Nrp1 RNAi (Fig. 2B). Thus, multiple Nrp1 siRNAs

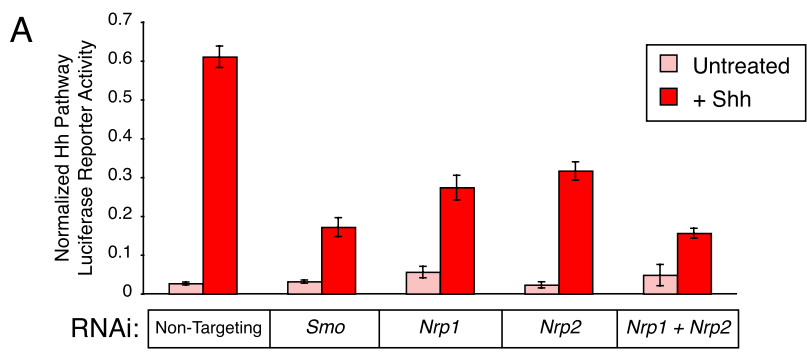

B

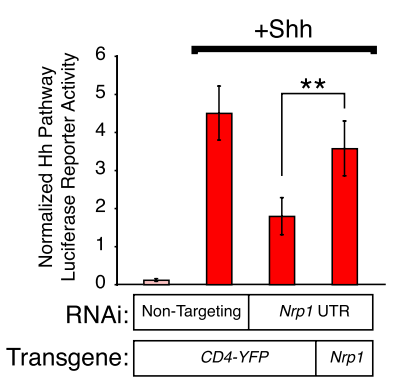

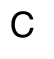

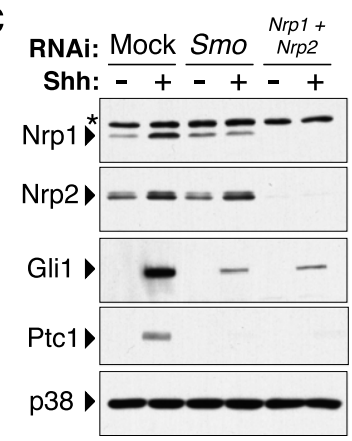

Figure 2. Nrp1 and Nrp2 are partially redundant positive regulators of $\mathrm{Hh}$ signal transduction. (A) Gli-dependent luciferase reporter (GLuc) transcription in NIH3T3 fibroblasts treated with Nrp1, Nrp2, or Nrp1+2 RNAi. $P<0.05$, two-tailed Student's $t$-test. Error bars indicate mean $\pm 1 \mathrm{SD}$. (B) GLuc transcription in NIH3T3 fibroblasts following Nrp1 3' UTR RNAi with or without coexpression of mouse Nrp1. $\left(^{\star \star}\right) P<0.01$, two-tailed Student's $t$-test. $(C)$ Immunoblots of Glil and Ptcl protein from NIH3T3 fibroblasts following Nrp1+2 RNAi and Shh treatment. The Nrp1 antibody detected Nrp1 and unrelated closely spaced nonspecific $\left({ }^{\star}\right)$ bands (Supplemental Fig. S1C).
(Supplemental Fig. 2A) inhibited Hh signal transduction and the inhibition could be specifically rescued, meeting the most stringent criteria for establishing the specificity of RNAi experiments (Cullen 2006). Rescue of the Nrp2 RNAi effect was more challenging, likely owing to extensive alternative splicing at this locus (Rossignol et al. 2000) and the existence of alternative 3' UTRs (Supplemental Fig. 2B). We instead confirmed the specificity of the Nrp2 RNAi effect by using multiple synthetic siRNAs targeting nonoverlapping Nrp2 sequence elements (Supplemental Fig. 2B). Four out of the five additional Nrp2 siRNAs tested caused significant Hh pathway inhibition (Supplemental Fig. 2C). Thus, in all, five separate RNAi reagents targeting Nrp2 produced strong Hh pathway inhibition, significantly reducing the likelihood that an off-target effect accounts for this phenomenon (Cullen 2006).

The luciferase reporter genes in Shh-LIGHT2 fibroblasts provide a faithful readout of Hh pathway activity, yet this system remains artificial and may in theory be subject to reporter-specific influences. We therefore sought an endogenous measurement of Hh pathway activity. The Gli1 and Ptc1 genes are strongly induced by Hh signaling in most cell types, making the Shh-stimulated increase in the abundance of these proteins an excellent metric of endogenous pathway activity (Ingham and McMahon 2001). We found that Nrp1+2 RNAi blocked Shh-stimulated Glil and Ptcl production, as assessed by immunoblot detection of these endogenous proteins (Fig. 2C). As expected, positive control RNAi against Smo also reduced the Shhstimulated increase in these two proteins. Antibodies to endogenous Nrp1 and Nrp2 confirmed the reduction in target protein abundance following Nrp1+2 RNAi treatment. (The Nrp1 antibody detected Nrp1 as well as a nonspecific band unrelated to Nrp1 gene products [Supplemental Fig. S1C].) Consistent with the immunoblot findings, Shh-stimulated accumulation of Gli1 and Ptc1 transcripts was reduced in cells treated with Nrp1+2 RNAi (Supplemental Fig. S3).

We next sought to test whether the inhibition of $\mathrm{Hh}$ signal transduction caused by Nrp1+2 RNAi was specific for the Hh pathway or was due to a more general derangement of intracellular signaling. If the latter were the case, we would expect other signaling modalities to be similarly affected by Nrp RNAi. Like the Hh pathway, the Wnt signaling pathway is an evolutionarily conserved mode of cell-cell communication (van Amerongen and Nusse 2009). Although Wnt and Hh signaling occur in proximity within many tissues during mammalian development, these pathways are comprised of largely distinct molecular components. To test whether Nrp regulation of Hh signaling is specific, we asked whether Nrp1 RNAi blocks canonical Wnt pathway signaling. We used an L-cell line stably transfected with a Wnt-responsive firefly luciferase reporter gene and a constitutive Renilla luciferase reporter gene (Hyman et al. 2009). We found that reporter gene expression stimulated by Wnt3A ligand was unaffected by Nrp1 RNAi, indicating that Nrp loss of function does not cause a general derangement of intracellular signal transduction (Supplemental Fig. S4A,B). Taken together, these data suggest that Nrps are specifically 
Hillman et al.

required by at least one step in the Hh signaling cascade in cultured fibroblasts.

To extend our in vitro studies, we next looked for Nrp expression in sites of active Hh signaling during mouse development. To do this, we stained mouse embryos at several developmental time points with antibodies capable of detecting endogenous Nrp1 and Smo. Cell populations with significant ciliary Smo protein were presumed to be undergoing active Hh signal transduction (Corbit et al. 2005; Rohatgi et al. 2007). The visceral yolk sac of the mouse embryo is comprised of an external layer of visceral endoderm and an internal, extraembryonic layer of yolk sac mesoderm. The yolk sac mesoderm gives rise to blood islands, which are sites of early hematopoiesis. Proper blood island formation is thought to occur in part as the result of an inductive Ihh signal from the visceral endoderm acting on yolk sac mesoderm (Becker et al. 1997; Dyer et al. 2001; Byrd et al. 2002). We observed Nrp1 production in cells of the yolk sac mesoderm in E8.5 mouse embryos (Fig. 3A). Cells in this layer also exhibited ciliary Smo, indicating active Hh signal transduction (Fig. 3B).

During development, Shh produced from the neural tube and notochord acts on adjacent paraxial mesoderm and is required for proper somite formation (Marigo and Tabin 1996; Marcelle et al. 1999; Resende et al. 2010). Genetic studies have demonstrated that this Shh signal is necessary for sclerotome induction (Chiang et al. 1996). We observed Nrp1 production in the paraxial mesoderm of E8.5 mouse embryos (Fig. 3C). We could tell that these Nrp1-expressing cells were undergoing active Hh signal transduction because they had high levels of ciliary Smo (Fig. 3D). Taken together, these data indicate that Nrp1 is present at several mesodermal locations of active $\mathrm{Hh}$ signal transduction in early mouse embryos, suggesting that Nrp1 may play a role in regulating Hh pathway output in these cell populations.

In E17.5 mouse skin, we identified Nrp1 and Nrp2 protein in dermal papillae of hair follicles and in the overlying epithelium (Fig. 3E). Murine hair follicles form at regular spatial intervals via the interplay of several epithelial-mesenchymal signals (Millar 2002). Shh from the follicle epithelium promotes epithelial proliferation and the formation of the underlying dermal papilla (StJacques et al. 1998; Chiang et al. 1999). Nrp1 protein was most abundant in the distal dermal condensate, whereas Nrp2 protein predominated in the proximal dermal condensate and overlying epithelium (Fig. 3E). Nrp1 staining colocalized with that for P75 neurotrophin receptor (P75NTR), a marker of the dermal condensate (Supplemental Fig. S5A,B).

To determine the functional contribution of Nrps to Hh signaling in the developing hair follicle, we used two lentiviruses to deliver shRNAs targeting $N r p 1+2$ to primary
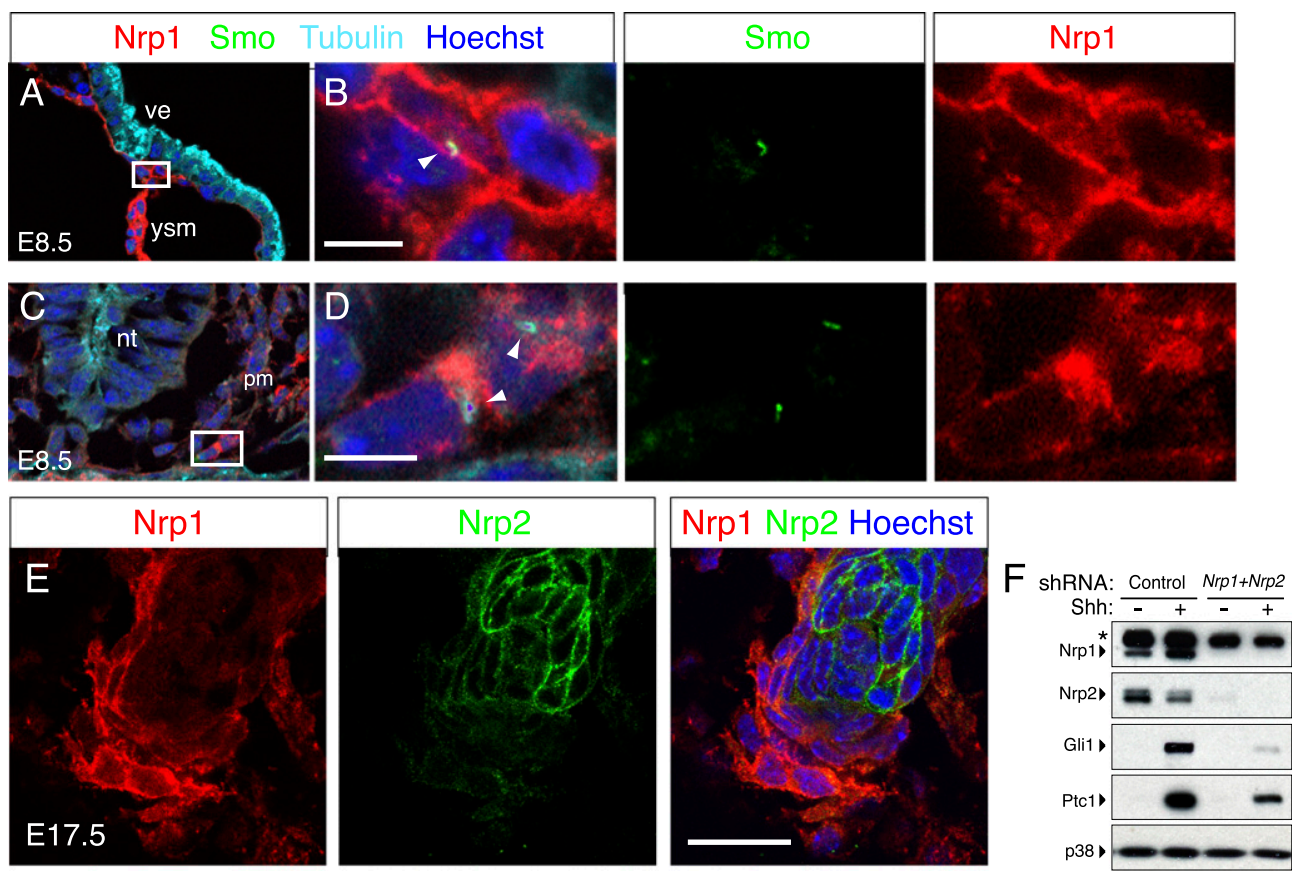

Figure 3. Nrps are expressed at locations of active Hh signaling during mouse development. (A) Fluorescence microscopy of Nrp1 (red, Alexa-595 labeled), Smo (green, Alexa-488-labeled), and acetylated tubulin (cyan, Alexa-633-labeled) expression in the visceral endoderm (ve) and yolk sac mesoderm (ysm) of E8.5 mouse embryos. (Blue) Hoechst dye-labeled nuclei. (B) Inset from image shown in $A$. Bar, $6 \mu \mathrm{m}$. (C) Fluorescence microscopy of Nrp1 (red, Alexa-595-labeled), Smo (green, Alexa-488 labeled), and acetylated tubulin (cyan, Alexa-633-labeled) expression in neural tube (nt) and paraxial mesoderm (pm) of E8.5 mouse embryos. (Blue) Hoechst dye-labeled nuclei. (D) Inset from image shown in C. Bar, $6 \mu \mathrm{m}$. (E) Fluorescence microscopy of Nrp1 (red, Alexa-595-labeled) and Nrp2 (green, Alexa-488-labeled) expression in the dermal papilla and epithelium of E17.5 mouse hair follicle. (Blue) Hoechst dye-labeled nuclei. Bar, $20 \mu \mathrm{m} .(F)$ Immunoblots of protein from Shh-stimulated primary dermal cells following infection with lentivirus expressing Nrp1+2 shRNAs. 
dermal cells isolated from newborn mice. These shRNAs targeted Nrp sequence elements that did not overlap with either the diced pools used in the initial screen or the synthetic siRNA reagents (Supplemental Fig. 2A,B). Shhstimulated induction of both Glil and Ptc1 proteins was strongly inhibited by Nrp1+2 shRNAs in the primary skin cultures (Fig. 3F). These results indicate that Nrps are present in cells undergoing $\mathrm{Hh}$ signal transduction at different stages of development and generalize our functional studies in NIH3T3 fibroblasts to a primary mesenchymal cell type that participates in complex Shhdependent morphogenesis in vivo.

\section{Nrp1 mediates a Hh pathway positive feedback circuit}

Transcription of genes encoding several Hh pathway components, including Gli1, Ptc1, and Hip, is increased in response to pathway activity (Goodrich et al. 1996; Lee et al. 1997; Chuang and McMahon 1999). Thus, transcriptional feedback loops are a common motif in Hh pathway regulation. During our initial investigations of Nrp function, we noted that the abundance of Nrp1 protein increased in Shh-treated NIH3T3 fibroblasts (Fig. 4A). This increase was approximately fivefold when averaged across independent experiments (Fig. 4B), in agreement with prior findings using endothelial cell lines (Hochman et al. 2006). Transcription of Nrp1 but not Nrp2 was increased in NIH3T3 fibroblasts treated with Shh, as measured by quantitative PCR (Supplemental Fig. 6A). Nrp1 transcription was also induced by Shh treatment in primary dermal cells prepared from postnatal mouse skin (Supplemental Fig. 6B). Shh-stimulated Nrp1 transcription was blocked by Smo RNAi (Fig. 4C), suggesting that this increase in Nrpl was a consequence of Hh pathway activation. In $\mathrm{Smo}^{-1-}$ mouse embryonic fibroblasts (MEFs), the abundance of Nrp1 was not increased in cells treated with either Shh or the small molecule $\mathrm{Hh}$ pathway agonist SAG (Fig. 4D), which acts by binding and activating Smo (Chen et al. 2002). When these MEFs were infected with a retrovirus encoding YFP-tagged Smo, accumulation of Nrpl protein could then be induced by Shh or SAG (Fig. 4D). Thus, the transcriptional induction of Nrp1 in response to Hh pathway activation requires Smo.

Several observations suggest that Nrp1 transcriptional induction by $\mathrm{Hh}$ signaling may not be directly mediated by Gli proteins. First, Nrp1 induction occurs after a significant delay. Nrp1 protein levels are not significantly changed until 8-12 h after Shh addition (Fig. 4E), in contrast to Ptc1, for which an appreciable rise can be detected as early as $4 \mathrm{~h}$ post-treatment (Fig. 4F). Second, the absolute fivefold induction of Nrp1 is significant yet smaller in magnitude than the direct Gli target, Ptc1 (Fig. 4F). Third, a previous Glil chromatin immunoprecipitation study conducted in our laboratory did not find evidence of Glil occupancy adjacent to the Nrp1 or Nrp2 promoters in
A

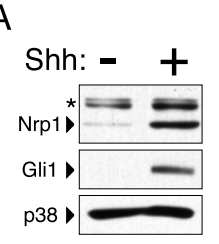

B

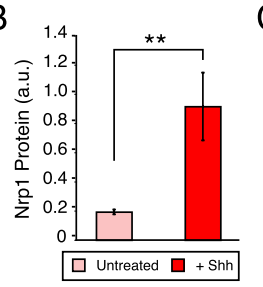

D

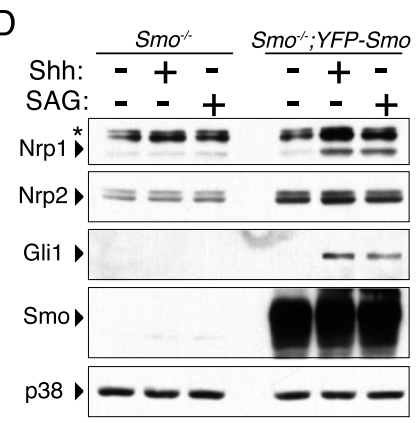

E



G

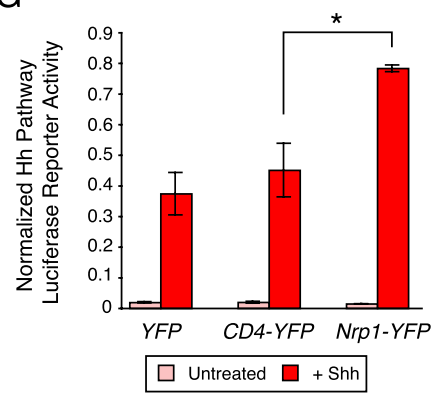

$\mathrm{F}$

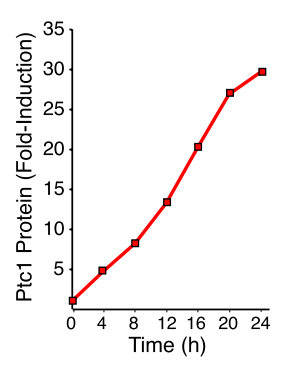

C

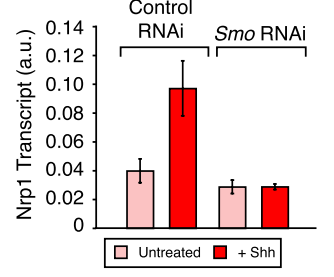

Figure 4. Nrpl mediates a Hh pathway positive feedback circuit. (A) Immunoblot of Nrpl protein abundance in NIH3T3 fibroblasts treated with Shh. (B) Quantitation of Nrp1 protein by densitometry of three independent immunoblots, normalized to p38 protein in the same lane. $\left(^{\star \star}\right) P<0.01$, two-tailed Student's $t$-test. Error bars indicate mean $\pm 1 \mathrm{SD} .(C)$ Quantitative PCR measurement of Shh-stimulated Nrp1 transcription following Smo RNAi. Units are PCR cycle thresholds normalized to those of Gapdh in the same well. Error bars indicate mean \pm 1 SD. (D) Immunoblots of protein from $\mathrm{Smo}^{-/-}$and rescued $\mathrm{Smo}^{-/-}$;YFP-Smo MEFs following Shh or SAG stimulation. $(E)$ Quantification of immunoblot showing Nrp1 protein expression as a function of time after addition of Shh. $(F)$ Quantification of immunoblot showing Ptc1 protein expression as a function of time after addition of Shh. (G) GLuc transcription in NIH3T3 fibroblasts overexpressing YFP, CD4-YFP, or Nrpl-YFP. $\left(^{\star}\right) P<0.05$, two-tailed Student's $t$-test. Measurements were normalized to a cotransfected constitutive Renilla luciferase. Error bars indicate mean \pm 1 SD. 
tumor cells with activated Hh signaling (Lee et al. 2010). Last, we found no Gli consensus binding sites conserved between the mouse and human Nrp1 promoter regions (see the Materials and Methods) using the rVista software tool (Loots et al. 2002), although Gli could possibly act from a distant enhancer. A similar analysis of the Nrp2 promoter revealed a single conserved sequence (aAACCACCCAga) with significant similarity to the canonical Gli-binding motif within $2 \mathrm{~kb}$ of the transcriptional start site, although this finding was of unclear significance as we did not observe transcriptional induction of $\mathrm{Nrp} 2$ in response to Shh. These data suggest that $\mathrm{Hh}$ pathway activation causes a rise in Nrp1 transcription that is likely mediated by an as-yet-unidentified intermediate transcription factor or factors. It will be of interest in the future to elucidate this transcriptional network in more detail and identify specific Nrp1 promoter sequence elements that mediate the response.

To test whether Nrpl produced in response to Shh feeds back to increase Hh target gene transcription, we fused the mouse Nrp1 coding region to that of YFP and transfected the expression vector into NIH3T3 fibroblasts. As a control, we fused the coding region of YFP to that of the CD4 receptor, a single-pass transmembrane protein comparable in molecular size with Nrps but otherwise entirely distinct (Supplemental Fig. S7A). Overexpression of Nrp1-YFP, but not CD4-YFP, significantly increased maximal Shh-stimulated transcription of a cotransfected Gli-dependent luciferase reporter (Fig. 4G). Under these conditions, Nrp1YFP was expressed at $\sim 10$-fold the level of endogenous Nrp1 protein (Supplemental Fig. S7B). Nrp1-YFP was able to rescue Hh pathway inhibition caused by Nrp1 RNAi to the same extent as an untagged Nrp1 cDNA (Supplemental Fig. S7C). Thus, we conclude that manipulation of Nrp1 protein concentration can positively or negatively modulate the transcriptional output of Hh pathway stimulation. Because Nrp1 is a target of $\mathrm{Hh}$ signaling, these data support the existence of a positive feedback circuit that may influence cell fates by modulating responsiveness to Hh ligands.

\section{Nrps act between Smo and SuFu to regulate Hh signal transduction}

Using molecular and genetic cell culture tools, we next investigated the step in the Hh pathway at which Nrps exert their influence. $\mathrm{Ptc1}^{-/-}$MEFs lack the inhibitory Hh receptor and consequently exhibit constitutive transcription of Hh pathway target genes such as Gli1 (Rohatgi et al. 2007; Humke et al. 2010). Nrp1+2 RNAi inhibited the high basal target gene activation in $\mathrm{Ptc1}^{-/-}$MEFs, as assayed by immunoblotting for Gli1 protein levels (Fig. 5A). Furthermore, Nrp1+2 RNAi blocked Shh-independent Ptc1 and Gli1 transcription caused by SAG (Fig. 5B). Overexpression of Nrp1-YFP sensitized cells to SAG (Fig. 5C) or to cotransfection of the constitutively active Smo-M2 mutant (Supplemental Fig. S8; Taipale et al. 2000; Chen et al. 2002). These data suggest that Nrp regulation of Hh signal transduction does not involve direct interaction with Ptc1 or Shh.
Nrps are well-characterized receptors for class 3 Semas, interacting with these ligands to promote growth cone collapse in certain neuronal cell populations (Chen et al. 1997; Kolodkin et al. 1997; Giger et al. 1998). One model of Nrp regulation of Hh signal transduction would involve cross-talk between a Sema signal and the Hh pathway. To test this idea, we titrated recombinant ShhN against a fixed, high concentration of Sema3A in the Shh-LIGHT2 reporter cell line. Across all ShhN concentrations tested, we did not observe positive or negative cross-regulation with Sema3A (Supplemental Fig. S9A). We also did not observe cross-regulation between ShhN and Sema3F (Supplemental Fig. S9C). Therefore, it is unlikely that a Sema-mediated signal is responsible for the regulation of Hh signal transduction by Nrps. Similarly, we did not observe cross-talk between Shh and the Nrp ligand VEGF164 (Supplemental Fig. S9B). Moreover, the Nrp coreceptor VEGF-R2 was not detected by antibodies in NIH3T3 fibroblasts (Supplemental Fig. S9D), even following extreme immunoblot overexposure (Supplemental Fig. S9D'). It appears, therefore, that the classical Nrp ligand families do not regulate Hh signal transduction through Nrps.

The primary cilium has a critical role in Hh signaling (Goetz and Anderson 2010). Binding of Shh to Ptcl causes Ptc1 to move out of cilia and Smo to move in (Corbit et al. 2005; Rohatgi et al. 2007). The loss of genes required for primary cilia to form or function can prevent regulation of target genes by Hh signals (Huangfu et al. 2003), an indirect but important influence that could be the basis for the Nrp effects. We found no defects in ciliation frequency or ciliary morphology in NIH3T3 fibroblasts treated with Nrp1+2 RNAi (Supplemental Fig. S10A). Shh-stimulated ciliary translocation of Smo was unaffected by Nrp1+2 RNAi (Supplemental Fig. S10B,C). Shh treatment still caused epitope-tagged HA-Gli2 to accumulate at the ciliary tip following Nrp1+2 RNAi (Supplemental Fig. S10D) in NIH3T3 fibroblasts engineered to express this transgene at low levels (Kim et al. 2010). Shh-stimulated accumulation of endogenous Gli2 was also unaffected by Nrp1+2 RNAi (Supplemental Fig. S10E). Thus, ciliation and the translocation of major Hh pathway components to this organelle are not dependent on the presence of Nrps.

A large fraction of cellular Nrp1 is present on the cell surface in NIH3T3 fibroblasts, as cells stained without detergent permeabilization exhibited robust Nrp1 immunofluorescence (Supplemental Fig. S10F). Despite the abundant surface localization, or perhaps because of it, we never observed a specific enrichment of Nrp1 in the primary cilium or significant alterations of the Nrp1 immunofluorescence pattern in response to Shh treatment (Supplemental Fig. 10C).

Smo controls Gli transcription factors by regulating $\mathrm{SuFu}$, an essential negative regulator of Hh signaling (Ingham and McMahon 2001). Nrp1+2 RNAi did not inhibit the high level of Glil protein accumulation that was observed in $\mathrm{SuFu}^{-/-}$MEFs (Fig. 5D), nor did overexpression of Nrp1-YFP potentiate Gli-dependent transcription of a luciferase reporter in $\mathrm{SuFu}^{-/-}$MEFs (Supplemental Fig. S11). Rescue of $\mathrm{SuFu}^{-1-} \mathrm{MEFs}$ with a SuFu-expressing retrovirus restored basal repression of Hh pathway target 
A

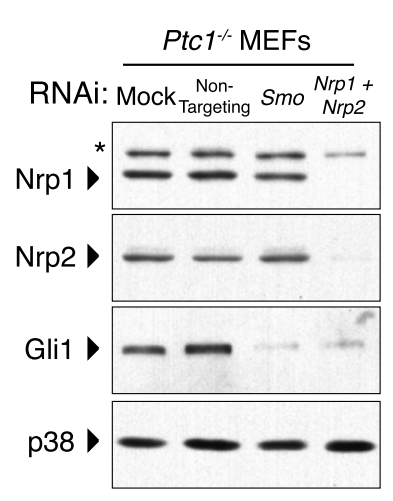

D

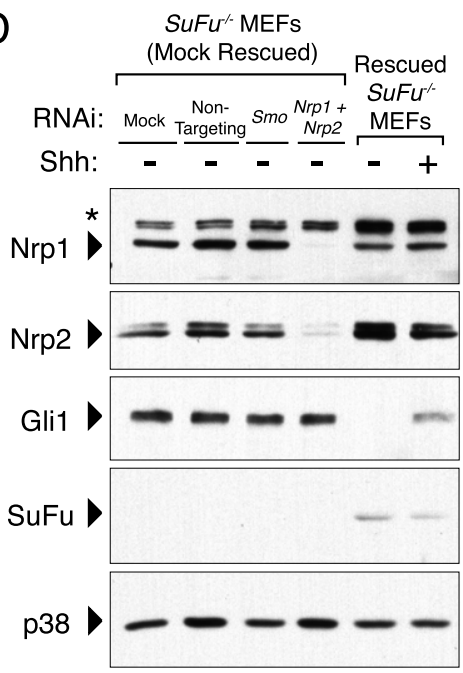

B

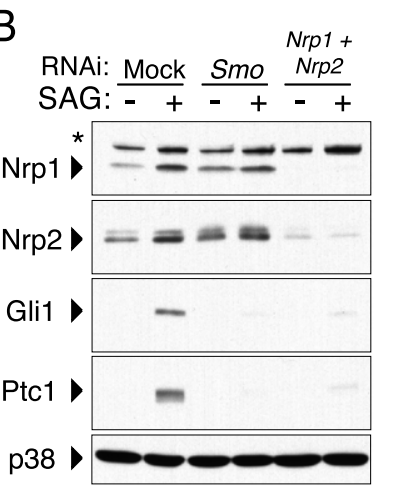

C

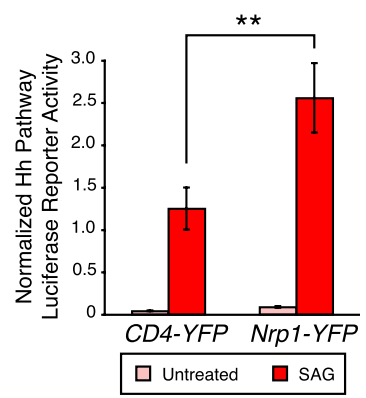

$E$

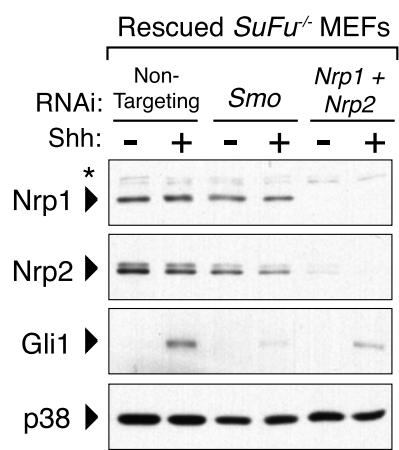

Figure 5. Nrps regulate $\mathrm{Hh}$ signal transduction between Smo and SuFu. $(A)$ Immunoblots of protein from unstimulated Ptc1 $1^{-1-}$ MEFs treated with Nrp1+2 RNAi. $(B)$ Immunoblots of protein from SAG-stimulated (100 nM) NIH3T3 fibroblasts treated with Nrp1+2 RNAi. (C) SAGstimulated GLuc transcription in NIH3T3 fibroblasts overexpressing Nrp1-YFP. $\left(^{\star \star}\right) P<0.01$, two-tailed Student's $t$-test. Measurements were normalized to a cotransfected constitutive Renilla luciferase. Error bars indicate mean $\pm 1 \mathrm{SD}$. $(D)$ Immunoblots of protein from unstimulated $\mathrm{SuFu}^{-1-}$ MEFs treated with $\mathrm{Nrp1+2}$ RNAi. Normal Shh responsiveness was restored in $\mathrm{SuFu}^{-/-} \mathrm{MEFs}$ infected with a retrovirus expressing SuFu ("Rescued") (Humke et al. 2010). (E) Immunoblots of protein from Shh-stimulated "Rescued" SuFu" ${ }^{-/}$MEFs treated with Nrp1+2 RNAi.

genes as well as responsiveness to stimulation by Shh (Fig. $5 \mathrm{E}_{;}$Humke et al. 2010). In these cells, Shh-stimulated pathway activation was sensitive to Nrp1+2 RNAi(Fig. 5E). The regulation of Hh signal transduction by Nrps therefore appears to be dependent on SuFu. These experiments also demonstrate that robust levels of $\mathrm{Hh}$ target gene expression can occur despite the loss of Nrps, suggesting the effect of Nrp RNAi is relatively specific to Hh pathway regulation and not the result of global cellular dysfunction.

\section{Nrps are conserved positive regulators of Hh signaling} in vivo

We next sought to determine whether Nrps are required for Hh signal transduction during embryogenesis in a living organism. Zebrafish possess four paralogous Nrp genes (nrp1a, nrp1b, nrp2a, and nrp2b), likely due to a gene duplication event since divergence from mammals (Supplemental Fig. S12). In zebrafish, Hh ligand produced in the notochord is essential for the specification of adaxial muscle pioneer cells that contribute to the formation of the horizontal myoseptum (Ingham and Kim 2005). Wildtype zebrafish embryos at $\sim 30 \mathrm{~h}$ post-fertilization (hpf) have straight bodies (Fig. 6A) and distinct chevron-shaped somites (Fig. 6B). Ptc1 is a Hh pathway target gene in fish, as it is in mice, and exhibits adaxial expression in bud stage (10 hpf) embryos (Fig. 6C). In contrast, shha is expressed in an axial pattern at this stage (Fig. 6D). Failure of Hh signaling to induce the horizontal myoseptum results in a distinctive phenotype characterized by ventral body curvature and U-shaped somites (van Eeden et al. 1996).

We injected individual antisense MOs targeting each zebrafish $n r p$ gene into one- to four-cell stage embryos. At low doses of a previously published translation-blocking nrp1a MO (MO1) (Lee et al. 2002), we observed tail vein defects that have been previously described, most obvious in the pooling of blood near the tail due to missing a vessel boundary separating the caudal artery and caudal vein between 24 and $48 \mathrm{hpf}$ (Martyn and Schulte-Merker 2004). At increased MO1 dosages (4 ng per embryo), besides the aforementioned vascular defects, nrp1a MO1 

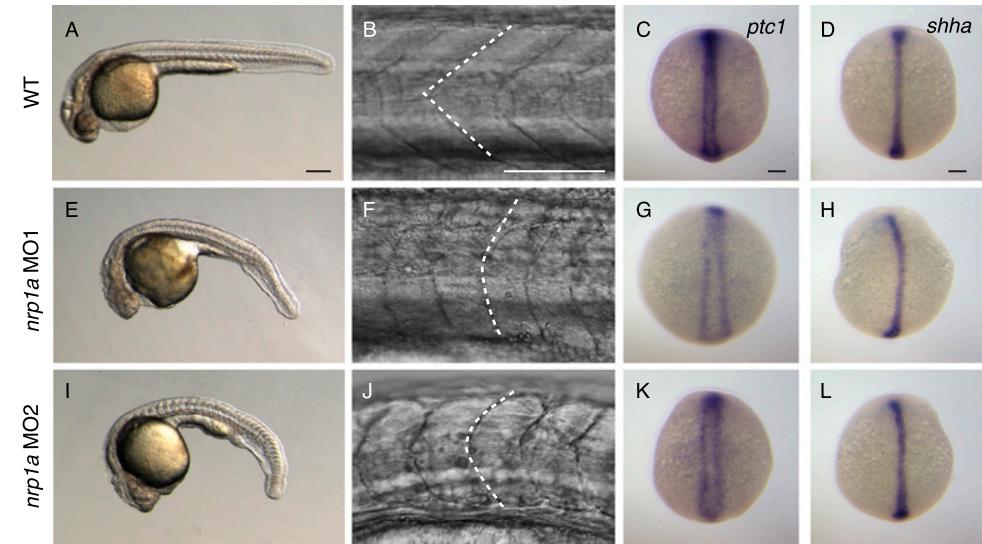
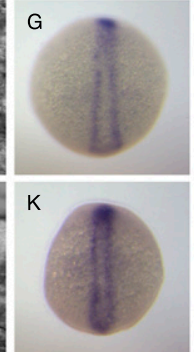

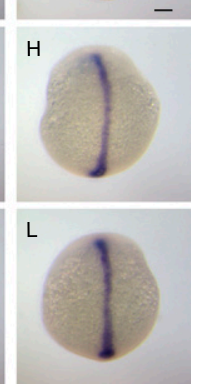

Figure 6. Zebrafish nrp1a morphants exhibit a Hh loss-of-function phenotype. $(A)$ Lateral view of wildtype zebrafish embryo at $\sim 30$ hpf. Bar, $100 \mu \mathrm{m}$. (B) Lateral view of chevron-shaped somites in wild-type zebrafish embryos at $\sim 30 \mathrm{hpf}$. Bar, $200 \mu \mathrm{m}$. (C) Wholemount in situ hybridization to ptc1 (purple) demonstrates adaxial staining pattern in bud stage (10 hpf) wild-type embryos. Bar, $200 \mu \mathrm{m}$. Dotted line delineates somite boundary. $(D)$ Whole-mount in situ hybridization to shha (purple) in wild-type $10 \mathrm{hpf}$ zebrafish embryos demonstrates axial expression. (E) At $\sim 30$ hpf, zebrafish embryos injected with nrp1a antisense MO1 at one- to four-cell stages exhibit ventral body curvature. $(F)$ Zebrafish nrp1a MO1-injected embryos exhibit U-shaped somites at $\sim 30 \mathrm{hpf}$. Dotted line delineate somite boundary. (G) Signal from ptc1 whole-mount in situ hybridization is significantly reduced in nrp1a MO1-injected embryos. (H) Signal from shha whole-mount in situ hybridization is unchanged in nrp1a MO1-injected embryos. (I) At $\sim 30$ hpf, zebrafish embryos injected with an orthogonal nrp1a antisense MO2 at one- to four-cell stages exhibit curved body morphology similar to nrp1a MO1 morphants. (J) Zebrafish nrp1a MO2-injected embryos exhibit U-shaped somites at 30 hpf, similar to nrp1a MO1 morphants. Dotted line delineates somite boundary. (K) Similar to ptc1 expression in nrp1a MO1 morphants, signal from ptc1 whole-mount in situ hybridization in nrp1a MO2-injected embryos is significantly reduced. $(L)$ Signal from shha whole-mount in situ hybridization is unchanged in nrp1a MO2-injected embryos. Views are dorsal, with anterior toward the top.

morphants exhibited a highly penetrant phenotype characterized by ventral body curvature (Fig. 6E) and U-shaped somites (Fig. 6F), consistent with the loss of adaxial $\mathrm{Hh}$ signal transduction (165 of 179 injected embryos). At the MO dosages we used, nrp1b, nrp2a, or nrp2b MOs did not result in obvious or consistent Hh loss-of-function phenotypes (data not shown). Adaxial ptc1 expression was reduced in 10 hpf nrp1a MO1 morphants (Fig. 6G), indicating reduced Hh signal transduction in receiving cells (37 of 39 injected embryos). The loss of ptc1 transcription was not due to loss of Hh ligand, because axial shha expression in nrp1a morphants (Fig. 6H) resembled that of wild-type animals (26 of 26 injected embryos).

To confirm the specificity of the Hh phenotype observed after nrp1a loss of function, we designed a second MO (nrp1a MO2) targeting a nonoverlapping nrp1a sequence element (see the Materials and Methods). Consistent with our previous findings, nrp1a MO2 morphants (4 ng per embryo) also exhibited ventral body curvature (Fig. 6I) and U-shaped somites (Fig. 6J), indicating a loss of adaxial Hh signal transduction (28 of 37 injected embryos). Adaxial ptc1 expression was reduced in 10 hpf nrp1a MO2 morphants (Fig. 6K), indicating loss of Hh signal transduction in receiving cells (16 of 19 injected embryos), while axial shha expression was unperturbed (nine of nine injected embryos) in nrp1a MO2 morphants (Fig. 6L). Thus, two MOs specifically targeted to nonoverlapping parts of zebrafish nrp1a mRNA produced specific and highly penetrant Hh pathway loss-of-function phenotypes. These data suggest that Nrps are essential positive regulators of embryonic Hh signaling in vivo, and that this role is phylogenetically conserved from mammals to bony fish.

\section{Discussion}

In this study, we identified Nrp1 in a focused mammalian Hh pathway RNAi screen and showed that Nrp1 and Nrp2 are partially redundant positive regulators of Hh signal transduction. The connection between Nrps and $\mathrm{Hh}$ signaling was unexpected. We selected Nrps for further investigation from among many strong hits in our RNAi screen in part because we were intrigued by the possibility that these well-characterized molecules, already known to regulate diverse signaling modalities, might have a previously unidentified role in Hh signal transduction.

\section{Multiple roles for Nrp proteins}

Earlier studies demonstrated Nrp genes are expressed at times and locations of active $\mathrm{Hh}$ signal transduction in the developing mouse nervous system (Kitsukawa et al. 1995; Chen et al. 1997). Our data demonstrate that Nrp1 and $\mathrm{Nrp} 2$ are expressed in mesodermal tissues undergoing active Hh signal transduction during mouse development. It is likely that the signaling role of Nrps differs among cell populations. Nrps are receptors for distinct ligands in neurons and endothelial cells, respectively (He and TessierLavigne 1997; Kolodkin et al. 1997; Soker et al. 1998). The cell-type specificity may depend in part on the local expression of Nrp coreceptors such as VEGF-R2/Kdr or plexins. Our data suggest that in mesodermal cell types, Nrps enhance Hh signal transduction and facilitate epithelial-mesenchymal interactions during development. Future experiments in mice or fish with appropriate genetic tools will be needed to fully address this question in vivo, but our fish data directly demonstrate the importance of Nrpla for Hh-dependent events in mesoderm development.

\section{Models for Nrp influences on Hedgehog transduction}

In this study, we investigated the mechanism of Nrp action in the Hh pathway to the extent allowed by our contemporary understanding of pathway biology. The molecular mechanisms of well-studied pathway components such 
as Smo and SuFu are only beginning to be understood, making it likely that a more detailed understanding of Nrp function will emerge concomitantly with increases in our understanding of Hh pathway biology. Nrps could affect Hh signal transduction in one or more of several distinct ways: action as a coreceptor for ligand or as a downstream transducer and integrator of external stimuli, or by affecting a basic cell property such as cilium formation, adhesion, or intracellular trafficking. Our experiments narrow the possibilities. First, we believe it is unlikely that Nrps control Hh reception by acting in a coreceptor capacity for Hh ligands, as activation of Hh signal transduction by ligand-independent methods (Ptc1 mutation, SAG stimulation) is sensitive to Nrp loss of function. Similarly, the inhibition of Hh signaling caused by Nrp RNAi is not simply due to generalized cellular derangement in fibroblasts, as canonical Wnt signaling is intact in the absence of Nrp function.

We also did not find evidence that Nrps act as signal integrators, because there appears to be no convergence between VEGF or Sema signals and the Hh pathway. In the cultured fibroblasts where we observed Hh signal transduction to require Nrp function, the Semas that interact with Nrp had no effect on $\mathrm{Hh}$ transduction and the VEGF receptor is not expressed. Possible interactions between $\mathrm{Hh}$ and these other pathways remain an open question for other cell types, but they do not explain the requirement for Nrps in cultured fibroblasts.

Primary cilia are required for $\mathrm{Hh}$ transduction, based on two combined lines of evidence. Mutations in components of cilia interfere with Hh transduction, and several pathway components are found located in cilia, some of them dynamically in response to ligand or to drugs that affect $\mathrm{Hh}$ transduction (Corbit et al. 2005; Rohatgi et al. 2007; Chen et al. 2009). Mutations that alter cilia lead to altered $\mathrm{Hh}$ transduction, so Nrp inhibition could affect cilia and, thus, Hh signals. We therefore monitored cilia structure after Nrp inhibition and saw no change in frequency or size of cilia. As a more precise measure of cilia function, we examined the trafficking of Smo and Gli2, two proteins whose concentration in cilia is a reflection of Hh ligand received. Both were unchanged following Nrp1+2 RNAi. Therefore, gross changes in cilia function or Hh pathway component localization are not responsible for the connection of Nrps to Hh transduction. As more is learned about how cilia process and transmit $\mathrm{Hh}$ transduction steps, additional tests of Nrp effects will be important. It is always possible that Nrps affect a subtle post-translational modification of a Hh pathway component that awaits elucidation.

Last, the mechanism of Nrp action could be to influence the Hh pathway by altering general cell properties. Overexpression of Nrp1 in fibroblasts can result in increased cell-cell adhesion, likely involving an interaction between Nrp1 and a second, unknown cell surface protein (Takagi et al. 1995). Subsequent work clarified the region of Nrp1 that mediates this adhesion but did not identify the putative interacting partner (Shimizu et al. 2000). We found that Nrp1 protein in NIH3T3 fibroblasts is present on the cell surface and exists predominantly outside the primary cilium. Nrp-mediated cell-cell adhesion could contribute to a cytoskeletal scaffold important for an as-yet-uncharacterized step in Hh signal transduction (Valdembri et al. 2009).

\section{The Nrp-Hedgehog positive feedback loop}

Multiple components of the Hh transduction machinery are regulated at the transcriptional level by the pathway itself. In the case of Ptc1, the boost of this negative regulator by a Hh signal potentially buffers the system. The induction of the gene encoding the surface Hh-binding protein Hip causes a phenotype like loss of Ihh, so this circuit also potentially restrains excess signaling (Chuang and McMahon 1999). The induction of Gli1 by $\mathrm{Hh}$ signals, in contrast, potentially serves as an amplifier. Nrp1 now joins the group of targets that affect the inducing pathway. Developmental cell fate specification by the Hh pathway is often quite dependent on the intensity and duration of the signal (Ribes and Briscoe 2009). We found that Nrp1 is transcriptionally activated by Hh signaling, but that this effect is unlikely to be mediated directly by Gli proteins. The Hh pathway gene regulatory network is clearly much larger than those genes that are direct targets of the Gli family of transcription factors, as genes encoding transcription factors such as $N$-myc and olig2 have been shown to be Hh pathway target genes in various tissue types (Oliver et al. 2003; Lee et al. 2010). A full characterization of this extended gene regulatory network will require significant work to elucidate, as it is difficult to globally predict transcription factor binding, and the subset of genes regulated by a particular transcription factor differs between tissue types. It will be interesting in the future to structurally analyze the Nrp1 enhancers and promoter to identify sequence elements required for transcriptional induction by $\mathrm{Hh}$ signaling. The sequence motifs present in this critical region of the Nrp1 promoter may suggest the identity of the transcription factor or factors that mediate Nrp1 transcriptional induction in response to Hh signaling. This could lead to the identification of an important secondary mediator of the Hh pathway transcriptional response.

Nrps act between Smo and Sufu, a part of the Hh pathway about which little is known. Converging upon that step are functions in cilia, multiple kinases, and several important trafficking events (Rohatgi and Scott 2007). It is likely that the partial redundancy of Nrp1 and Nrp2 in this capacity has in the past obscured their role as Hh pathway regulators in vivo. A recent study demonstrated that pretreatment of spinal cord commissural axons with Shh sensitized them to repulsive Sema cues (Parra and Zou 2010). Whether transcriptional cross-regulation of Nrp expression by Shh occurs in neuronal cell populations or other sites of Hh signaling in vivo is an important topic for further inquiries. Nrps have, in recent times, gained interest as targets for cancer therapeutics due to their important roles in VEGF signaling and, consequently, the supply of oxygen to growing tumors. The involvement of Nrps in Hh signal transduction raises the possibility that some Nrp-based interventions could interfere with tumor growth through their effects on multiple signaling pathways. 
Hillman et al.

\section{Materials and methods}

Cell culture

NIH3T3 fibroblasts were obtained from American Type Culture Collection. Shh-LIGHT2 cells were from a previously described stock (Taipale et al. 2000). Ptc1 ${ }^{-1-}$ MEFs were derived as previously described (Rohatgi et al. 2007). SuFu ${ }^{-/-}$MEFs transduced with empty retrovirus and SuFu-DD retrovirus, as well as rescued $\mathrm{Smo}^{-1-} \mathrm{MEFs}$, were a gift from Rajat Rohatgi (Rohatgi et al. 2009; Humke et al. 2010). The HA-Gli2 was a gift from the Beachy laboratory (Kim et al. 2009). The Wnt luciferase reporter cells were from a previously described stock (Hyman et al. 2009). All cell lines were maintained in complete DMEM supplemented with 10\% fetal bovine serum (FBS) unless otherwise indicated. For Shh or SAG treatment, NIH3T3 fibroblasts, MEFs, and primary dermal cells were grown to confluency and switched to DMEM supplemented with $0.5 \%$ FBS to promote ciliation (Rohatgi et al. 2007; Wen et al. 2010). Agonist treatment durations were 24-30 h unless otherwise noted.

\section{RNAi screen}

DSPs were produced and purified using previously published methods (Myers et al. 2003; Galvez et al. 2007). DSPs were introduced into Shh-LIGHT2 cells (Taipale et al. 2000) via a "wet" reverse transfection procedure. Cells were grown to confluency $(\sim 24 \mathrm{~h})$ then switched into complete 0.5\% FBS DMEM conditioned with ShhN, the active moiety of Shh ligand /Chen et al. 2002). After 24-30 h of Shh stimulation, cells were lysed and firefly luciferase and Renilla luciferase signals were read using the Dual-Glo system (Promega). Firefly/Renilla ratios were normalized to the average ratio of the negative control wells on the same plate to control for plate-to-plate and day-to-day assay variability. DSPs that reduced the mean Renilla signal $>30 \%$ compared with negative control DSPs on each plate were removed from further analysis, as they likely affected cell survival or proliferation. The mean normalized values for replicate wells were calculated and converted to Z-scores using the mean and standard deviation of all Renilla-filtered wells. A Z-score cutoff of \pm 1.5 was chosen as a significance threshold.

\section{Synthetic RNAi reagents}

Mouse Nrp1 siRNA (\#1, 5'-GCACAAAUCUCUGAAACUA-3'; Dharmacon), mouse Nrp2 siRNA (\#1, 5'-GACAAUGGCUGGA CACCCA-3' ; Sigma), mouse Smo siRNA (SASI_Mm01_00346929, Sigma), and nontargeting siRNAs (Dharmacon) were dissolved in nuclease-free water and stored as $5 \mu \mathrm{M}$ stocks. A custom siRNA (denoted \#2, 5' - GCUCUGAAGACCUGGCAAUUU-3'; Dharmacon) targeting the mouse Nrp1 3' UTR was used for cDNA rescue experiments. Custom siRNAs targeting the mouse Nrp2 3' UTR (denoted \#2, 5' -GGAUAUAAGUGCAAAGACA-3'; denoted \#3, 5'-UAACAAAGGAAGAGAGAGA-3'; denoted \#4, 5'-GCACAGUGGUAGAGGUGAA-3'; denoted \#5, 5'-GAG CAGAGAGAAAGAAUAA-3'; and denoted \#6, 5'-GAAAUU GUGUGAAGGAUAA-3 ${ }^{\prime}$ ) were designed and purchased from Dharmacon.

\section{Transient transfections}

In all cases, siRNAs were transiently introduced using a "wet" reverse transfection procedure in either 96-well, eight-chamber slide, or 60-mm plate format. For siRNAs, Lipofectamine 2000 (Invitrogen) was used in NIH3T3 fibroblasts and Shh-LIGHT2 cells. Dharmafect 4 (Dharmacon) was used for MEF transfections.
Plasmid DNA was transfected using Fugene6 (Roche). For overexpression assays, transgene DNA was introduced along with a Gli-dependent luciferase/TK-Renilla reporter plasmid mixture. Rescue experiments were carried out sequentially with reverse transfection of 3' UTR siRNA on day 1, dropwise cDNA and luciferase reporter cotransfection on day 2 , addition of Shh and serum starvation on day 3 , and harvest on day 4.

\section{Immunoblotting}

Cells were scraped into cold phosphate-buffered saline (PBS), sedimented at $1000 \mathrm{~g}$ for $5 \mathrm{~min}$, and lysed in a modified RIPA buffer (25 mM Na-Tris at pH 7.4, $150 \mathrm{mM} \mathrm{NaCl}, 2 \%$ [v/v] NP-40, $0.25 \%[\mathrm{w} / \mathrm{v}]$ sodium deoxycholate, $1 \mathrm{mM}$ DTT, $1 \mathrm{mM}$ PMSF, Roche Complete protease inhibitor cocktail with EDTA) for 30$60 \mathrm{~min}$ at $4^{\circ} \mathrm{C}$. The lysate was clarified by centrifugation at $20,000 \mathrm{~g}$ for $30 \mathrm{~min}$. Protein concentrations of the supernatants were determined using the detergent-insensitive BCA kit (Pierce). Equal amounts of total protein from the samples were supplemented with SDS buffer (final composition: $50 \mathrm{mM} \mathrm{Na}$-Tris at $\mathrm{pH}$ $6.8,2 \%$ SDS, $6 \%$ glycerol, $1 \% \beta$-mercaptoethanol), incubated for $15 \mathrm{~min}$ at room temperature, resolved by SDS-PAGE, and then processed for immunoblotting. Samples were not boiled prior to electrophoresis. Anti-p38 (1:50,000; Sigma), anti-HA (Covance), anti-Gli1 (1:500; Cell Signaling Technologies), anti-Gli2 (1:200; R\&D Systems), anti-Vegfr2/KDR (1:1000; Cell Signaling Technologies), anti-Nrp1 (1:1000; Cell Signaling Technologies), and antiNrp2 (1:1000; Cell Signaling Technologies) were purchased from various vendors. Anti-Ptc1 (1:500) (Rohatgi et al. 2007), anti-Smo (1:500) (Rohatgi et al. 2007), and anti-SuFu (1:2500) (Humke et al. 2010) were raised as previously described. All primary antibody incubations were carried out overnight at $4^{\circ} \mathrm{C}$ in $5 \%$ nonfat dry milk Tris-buffered saline (pH 7.4) containing 0.05\% Tween-20.

\section{Cell immunofluorescence}

Smo and Nrp1 were detected in fixed samples by immunofluorescence using anti-Smo, anti-acetylated tubulin, and anti-Nrp1 with nuclei stained using Hoescht dye. NIH3T3 fibroblasts were reverse-transfected with siRNAs on eight-chamber slides (LabTek) using a scaled version of the procedure described above for 96-well plates. After culturing and treatment, cells were fixed with $4 \%$ paraformaldehyde (PFA) in PBS for $15 \mathrm{~min}$ and washed three times with PBS. Fixed cells were placed in blocking solution (PBS with $1 \%[\mathrm{v} / \mathrm{v}]$ normal donkey serum, $0.1 \%[\mathrm{v} / \mathrm{v}]$ Triton X-100) for $30 \mathrm{~min}$. Primary antibodies (anti-Nrp1 [1:500; R\&D Systems], anti-Smo [1:500] [Rohatgi et al. 2007], and antiacetylated tubulin [1:2000] [Sigma]) were diluted in block and used to stain cells for 1 $\mathrm{h}$ at room temperature. After washing three times in PBS, Alexa dye-coupled secondary antibodies were added in block solution at 1:500 for $1 \mathrm{~h}$ at room temperature. Hoescht dye was included in the final washes before the samples were mounted in Fluoromount $\mathrm{G}$ (Southern Biotech) for microscopy.

\section{Tissue histology}

Frozen sections of E8.5 and E16.5-E17.5 mouse embryos were fixed in PFA, then permeablized in a $5 \%$ NHS/Triton $0.05 \%$ block solution. Primary antibodies (rat anti-CD31 [1:25; BD Pharmingen], rabbit anti-P75NTR [1:200; Abcam], goat anti-Nrp1 [1:100; R\&D Systems], rabbit anti-Nrp2 [1:1000; Cell Signaling], rabbit anti-Smo [1:500] [Rohatgi et al. 2007], and mouse anti-acetylated tubulin [1:2000; Sigma]) were diluted in block solution. Alexa dyecoupled secondary antibodies were used in block solution at 1:500. Hoescht dye was used to highlight nuclei. 


\section{Lentivirus production}

Hairpin sequences targeting mouse $\mathrm{Nrp} 1\left(\mathrm{H}, 5^{\prime}\right.$-GGAGATGA GAAGATAGTAA-3') or Nrp2 (H, 5'-GAACTGGAGAGAACA TACA-3') were cloned into the pSicoR vector according to standard protocols (http://web.mit.edu/jacks-lab/protocols_table. html; Ventura et al. 2004). Lentivirus was generated in 293T cells by cotransfecting $\Delta 8.9$ and VSVG packing vectors with the pSicoR shRNA vector. After $20 \mathrm{~h}$, the culture medium was replaced, and viral supernatant was collected at $48 \mathrm{~h}$ post-transfection, filtered, and frozen.

\section{Dermal cell isolation and infection}

Primary dermal cells were isolated as previously described (Lichti et al. 2008). Briefly, we dissected mouse skin from pups within $3 \mathrm{~d}$ of birth. We used dispase (dispase II, Roche) to separate dermis from epidermis. To dissociate dermal cells from dermis, we incubated minced dermis with $0.25 \%$ collagenase (Sigma) for $45 \mathrm{~min}$ at $37^{\circ} \mathrm{C}$. After neutralizing with FBS, we filtered the digested dermis through a $70-\mu \mathrm{m}$ cell strainer (BD Falcon), spun it at low speed $(30 g)$, collected the supernatant, and spun it at $200 \mathrm{~g}$ to collect single dermal cells. We typically recovered $2 \times$ $10^{7}$ dermal cells per pup skin. We infected the primary dermal cells at $50 \%$ confluence $1 \mathrm{~d}$ after plating using $8 \mu \mathrm{g} / \mathrm{mL}$ polybrene as carrier and spun the cells at $1000 \mathrm{rpm}$ for an hour, then replaced it with fresh medium. Upon reaching confluency, cells were switched into 0.5\% DMEM conditioned with Shh and incubated for an additional $30 \mathrm{~h}$. All experiments were performed in primary dermal cells without passage.

\section{Microscopy}

Fixed cell microscopy was performed on an inverted Leica DMIRE2 laser-scanning confocal microscope. Images were taken with a $63 \times$ objective and $4 \times$ zoom.

\section{Image analysis}

All analyses were performed in the program ImageJ, as previously described (Rohatgi et al. 2007). To quantitate the fluorescent signal of Smo in primary cilia, a mask was constructed by manually outlining cilia in the image taken in the acetylated tubulin channel. This mask was applied to the image taken in the Smo channel and the fluorescence at cilia was measured. Local background correction was performed by moving the mask to measure fluorescence at a representative nearby region; this value was subtracted from that of ciliary fluorescence.

\section{Quantitative PCR}

Total RNA was isolated from NIH3T3 fibroblasts using Trizol reagent (Invitrogen). One microgram of RNA was reverse-transcribed with random hexamer primers using SuperScript III reverse transcriptase (Invitrogen). A fraction (1/20) of the resultant cDNA was used as a template for interrogation with TaqMan quantitative PCR probes (Applied Biosystems) on an Applied Biosystems 7500 Fast thermocycler: Gapdh (Mm99999915_g1), Gli1 (Mm00494645_m1), Ptc1 (Mm00436026_m1), Nrp1 (Mm00435371_m1), and Nrp2 (Mm00803099_m1).

\section{DNA constructs}

Mouse Nrp1 and CD4 cDNAs were obtained from Open Biosystems. The ORF of each was PCR-amplified and cloned in-frame into pEYFPN1 (Clontech). To generate the untagged
Nrp1 vector used in the RNAi rescue experiments, a stop codon was introduced by PCR mutagenesis into the Nrp1-YFP vector following the Nrp1 ORF. Gli-luciferase and TK-Renilla plasmids have been previously described (Taipale et al. 2000; Humke et al. 2010).

\section{Gli motif identification}

The rVista (Loots et al. 2002) conserved transcription factorbinding site prediction tool was accessed through the zPicture online interface (http://zpicture.dcode.org). The $10 \mathrm{~kb}$ of genomic sequence upstream of the mouse (mm9) and human (hg19) Nrp1 or Nrp2 transcriptional start sites were used in this analysis. Default parameters were used in all cases.

\section{Pathway agonists}

Medium conditioned with active, $\mathrm{N}$-terminal ShhN ligand was produced using a HEK 293 line that stably secretes this protein, as previously described (Chen et al. 2002). Wnt3A conditioned medium was produced in a similar manner from a separate HEK 293 line. SAG was obtained from Enzo Life Sciences.

\section{Recombinant proteins}

Recombinant mouse ShhN, mouse VEGF ${ }_{164}$, and human Sema3A/ Fc chimera were obtained from R\&D Systems and dissolved in PBS containing $0.1 \%$ BSA.

\section{Zebrafish strain and maintenance}

Wild-type zebrafish (Danio rerio) used AB background and were raised and maintained under standard conditions.

\section{Phylogenetic tree}

A phylogenetic tree was produced by a multiple alignment of zebrafish, mouse, and human neuroplin proteins by T-Coffee (http://tcoffee.vital-it.ch/cgi-bin/Tcoffee/tcoffee_cgi/index.cgi) and distance matrix computation by PHYLIP protdist (http://mobyle. pasteur.fr/cgi-bin/portal.py?form=bionj). The phylogram was drawn with the program Newicktops (http://mobyle.pasteur.fr/cgi-bin/ portal.py?form=bionj).

\section{Whole-mount RNA in situ hybridization}

Whole-mount RNA in situ hybridization of zebrafish embryos was performed according to standard protocols (Thisse and Thisse 2008). Digoxigenin (DIG)-RNA probes were generated according to the manufacturer's instructions (Roche). The DIGlabeled probes included those previously published for ptc1 and shha (Concordet et al. 1996).

\section{MO knockdown}

Antisense MOs (GeneTools) were microinjected into one- to fourcell stage embryos according to standard protocols (Nasevicius and Ekker 2000). For the experiments reported in Figure 6, $4 \mathrm{ng}$ of MO was used per injection. MO sequences used were nrp1aMO1 (5'-GAATCCTGGAGTTCGGAGTGCGGAA-3') (Lee et al. 2002); $n r p 1 a-M O 2$ (5'-TGGCAAAAAACGATGAGACAATCCT-3'); and $r p 1 b$-MO $\left(5^{\prime}\right.$-CCAGTACATCCTCAAACGAAATC-3'), nrp2aMO (5'-TATCCAGAAATCCATCTTTCCG- $\left.3^{\prime}\right)$, and $n r p 2 b-M O$ (5'-GCGAATAAATCCATCTTTCCTG-3') from Martyn and Schulte-Merker (2004). 


\section{Acknowledgments}

We thank R. Rohatgi, E. Humke, E. Lee, and A. Zhang for discussions, ideas, and reagents. R.T.H. is supported by Stanford University's Medical Scientist Training Program and a Bio-X Graduate Fellowship. B.Y.F. is supported by an NIH KirschsteinNRSA post-doctoral fellowship (F32CA134146). M.G.H.G. is supported by a post-doctoral fellowship from the California Institute of Regenerative Medicine (TG2-01159). J.K.C. gratefully acknowledges support from the NIH/NCI (R01 CA136574), and A.E.O. gratefully acknowledges support from NIH/NIAMS (R01 ARO46786). The work was supported by NIH grant RO1GM095948 to M.P.S. M.P.S. is an Investigator of the Howard Hughes Medical Institute.

\section{References}

Bai CB, Joyner AL. 2001. Glil can rescue the in vivo function of Gli2. Development 128: 5161-5172.

Beachy PA, Karhadkar SS, Berman DM. 2004. Tissue repair and stem cell renewal in carcinogenesis. Nature 432: 324-331.

Becker S, Wang ZJ, Massey H, Arauz A, Labosky P, Hammerschmidt M, St-Jacques B, Bumcrot D, McMahon A, Grabel L. 1997. A role for Indian hedgehog in extraembryonic endoderm differentiation in F9 cells and the early mouse embryo. Dev Biol 187: 298-310.

Byrd N, Becker S, Maye P, Narasimhaiah R, St-Jacques B, Zhang X, McMahon J, McMahon A, Grabel L. 2002. Hedgehog is required for murine yolk sac angiogenesis. Development 129: 361-372.

Charron F, Stein E, Jeong J, McMahon AP, Tessier-Lavigne M. 2003. The morphogen sonic hedgehog is an axonal chemoattractant that collaborates with netrin-1 in midline axon guidance. Cell 113: 11-23.

Chen H, Chedotal A, He Z, Goodman CS, Tessier-Lavigne M. 1997. Neuropilin-2, a novel member of the neuropilin family, is a high affinity receptor for the semaphorins Sema E and Sema IV but not Sema III. Neuron 19: 547-559.

Chen H, Bagri A, Zupicich JA, Zou Y, Stoeckli E, Pleasure SJ, Lowenstein DH, Skarnes WC, Chedotal A, Tessier-Lavigne M. 2000. Neuropilin-2 regulates the development of selective cranial and sensory nerves and hippocampal mossy fiber projections. Neuron 25: 43-56.

Chen JK, Taipale J, Young KE, Maiti T, Beachy PA. 2002. Small molecule modulation of Smoothened activity. Proc Natl Acad Sci 99: 14071-14076.

Chen MH, Wilson CW, Li YJ, Law KK, Lu CS, Gacayan R, Zhang X, Hui CC, Chuang PT. 2009. Cilium-independent regulation of Gli protein function by Sufu in Hedgehog signaling is evolutionarily conserved. Genes Dev 23: 1910-1928.

Cheng HJ, Bagri A, Yaron A, Stein E, Pleasure SJ, Tessier-Lavigne M. 2001. Plexin-A3 mediates semaphorin signaling and regulates the development of hippocampal axonal projections. Neuron 32: 249-263.

Chiang C, Litingtung Y, Lee E, Young KE, Corden JL, Westphal H, Beachy PA. 1996. Cyclopia and defective axial patterning in mice lacking Sonic hedgehog gene function. Nature 383: 407-413.

Chiang C, Swan RZ, Grachtchouk M, Bolinger M, Litingtung Y, Robertson EK, Cooper MK, Gaffield W, Westphal H, Beachy PA, et al. 1999. Essential role for Sonic hedgehog during hair follicle morphogenesis. Dev Biol 205: 1-9.

Chuang PT, McMahon AP. 1999. Vertebrate Hedgehog signalling modulated by induction of a Hedgehog-binding protein. Nature 397: 617-621.

Concordet JP, Lewis KE, Moore JW, Goodrich LV, Johnson RL, Scott MP, Ingham PW. 1996. Spatial regulation of a zebrafish patched homologue reflects the roles of sonic hedgehog and protein kinase A in neural tube and somite patterning. Development 122: 2835-2846.

Corbit KC, Aanstad P, Singla V, Norman AR, Stainier DY, Reiter JF. 2005. Vertebrate Smoothened functions at the primary cilium. Nature 437: 1018-1021.

Cullen BR. 2006. Enhancing and confirming the specificity of RNAi experiments. Nat Methods 3: 677-681.

Dyer MA, Farrington SM, Mohn D, Munday JR, Baron MH. 2001. Indian hedgehog activates hematopoiesis and vasculogenesis and can respecify prospective neurectodermal cell fate in the mouse embryo. Development 128: 1717-1730.

Galvez T, Teruel MN, Heo WD, Jones JT, Kim ML, Liou J, Myers JW, Meyer T. 2007. siRNA screen of the human signaling proteome identifies the PtdIns $(3,4,5)$ P3-mTOR signaling pathway as a primary regulator of transferrin uptake. Genome Biol 8: R142. doi: 10.1186/gb-2007-8-7-r142.

Geretti E, Shimizu A, Klagsbrun M. 2008. Neuropilin structure governs VEGF and semaphorin binding and regulates angiogenesis. Angiogenesis 11: 31-39.

Giger RJ, Urquhart ER, Gillespie SK, Levengood DV, Ginty DD, Kolodkin AL. 1998. Neuropilin-2 is a receptor for semaphorin IV: Insight into the structural basis of receptor function and specificity. Neuron 21: 1079-1092.

Giger RJ, Cloutier JF, Sahay A, Prinjha RK, Levengood DV, Moore SE, Pickering S, Simmons D, Rastan S, Walsh FS, et al. 2000. Neuropilin-2 is required in vivo for selective axon guidance responses to secreted semaphorins. Neuron 25: 29-41.

Goetz SC, Anderson KV. 2010. The primary cilium: A signalling centre during vertebrate development. Nat Rev Genet 11: 331-344.

Goodrich LV, Johnson RL, Milenkovic L, McMahon JA, Scott MP. 1996. Conservation of the hedgehog/patched signaling pathway from flies to mice: Induction of a mouse patched gene by Hedgehog. Genes Dev 10: 301-312.

He Z, Tessier-Lavigne M. 1997. Neuropilin is a receptor for the axonal chemorepellent Semaphorin III. Cell 90: 739-751.

Hochman E, Castiel A, Jacob-Hirsch J, Amariglio N, Izraeli S. 2006. Molecular pathways regulating pro-migratory effects of Hedgehog signaling. J Biol Chem 281: 33860-33870.

Huangfu D, Liu A, Rakeman AS, Murcia NS, Niswander L, Anderson KV. 2003. Hedgehog signalling in the mouse requires intraflagellar transport proteins. Nature 426: 83-87.

Hui CC, Joyner AL. 1993. A mouse model of greig cephalopolysyndactyly syndrome: The extra-toesJ mutation contains an intragenic deletion of the Gli3 gene. Nat Genet 3: 241-246.

Humke EW, Dorn KV, Milenkovic L, Scott MP, Rohatgi R. 2010. The output of Hedgehog signaling is controlled by the dynamic association between Suppressor of Fused and the Gli proteins. Genes Dev 24: 670-682.

Hyman JM, Firestone AJ, Heine VM, Zhao Y, Ocasio CA, Han K, Sun M, Rack PG, Sinha S, Wu JJ, et al. 2009. Small-molecule inhibitors reveal multiple strategies for Hedgehog pathway blockade. Proc Natl Acad Sci 106: 14132-14137.

Ingham PW, Kim HR. 2005. Hedgehog signalling and the specification of muscle cell identity in the zebrafish embryo. Exp Cell Res 306: 336-342.

Ingham PW, McMahon AP. 2001. Hedgehog signaling in animal development: Paradigms and principles. Genes \& Dev 15: 3059-3087.

Kawasaki T, Kitsukawa T, Bekku Y, Matsuda Y, Sanbo M, Yagi T, Fujisawa H. 1999. A requirement for neuropilin-1 in embryonic vessel formation. Development 126: 4895-4902.

Kim J, Kato M, Beachy PA. 2009. Gli2 trafficking links Hedgehog-dependent activation of Smoothened in the primary 
cilium to transcriptional activation in the nucleus. Proc Natl Acad Sci 106: 21666-21671.

Kim J, Lee JJ, Gardner D, Beachy PA. 2010. Arsenic antagonizes the Hedgehog pathway by preventing ciliary accumulation and reducing stability of the Gli2 transcriptional effector. Proc Natl Acad Sci 107: 13432-13437.

Kitsukawa T, Shimono A, Kawakami A, Kondoh H, Fujisawa H. 1995. Overexpression of a membrane protein, neuropilin, in chimeric mice causes anomalies in the cardiovascular system, nervous system and limbs. Development 121: 43094318.

Kolodkin AL, Levengood DV, Rowe EG, Tai YT, Giger RJ, Ginty DD. 1997. Neuropilin is a semaphorin III receptor. Cell 90: 753-762.

Lee J, Platt KA, Censullo P, Ruiz i Altaba A. 1997. Glil is a target of Sonic hedgehog that induces ventral neural tube development. Development 124: 2537-2552.

Lee P, Goishi K, Davidson AJ, Mannix R, Zon L, Klagsbrun M. 2002. Neuropilin-1 is required for vascular development and is a mediator of VEGF-dependent angiogenesis in zebrafish. Proc Natl Acad Sci 99: 10470-10475.

Lee EY, Ji H, Ouyang Z, Zhou B, Ma W, Vokes SA, McMahon AP, Wong WH, Scott MP. 2010. Hedgehog pathway-regulated gene networks in cerebellum development and tumorigenesis. Proc Natl Acad Sci 107: 9736-9741.

Lichti U, Anders J, Yuspa SH. 2008. Isolation and short-term culture of primary keratinocytes, hair follicle populations and dermal cells from newborn mice and keratinocytes from adult mice for in vitro analysis and for grafting to immunodeficient mice. Nat Protoc 3: 799-810.

Loots GG, Ovcharenko I, Pachter L, Dubchak I, Rubin EM. 2002. rVista for comparative sequence-based discovery of functional transcription factor binding sites. Genome Res 12: 832-839.

Marcelle C, Ahlgren S, Bronner-Fraser M. 1999. In vivo regulation of somite differentiation and proliferation by Sonic Hedgehog. Dev Biol 214: 277-287.

Marigo V, Tabin CJ. 1996. Regulation of patched by sonic hedgehog in the developing neural tube. Proc Natl Acad Sci 93: 9346-9351.

Martyn U, Schulte-Merker S. 2004. Zebrafish neuropilins are differentially expressed and interact with vascular endothelial growth factor during embryonic vascular development. Dev Dyn 231: 33-42.

Millar SE. 2002. Molecular mechanisms regulating hair follicle development. I Invest Dermatol 118: 216-225.

Myers JW, Jones JT, Meyer T, Ferrell JE Jr. 2003. Recombinant Dicer efficiently converts large dsRNAs into siRNAs suitable for gene silencing. Nat Biotechnol 21: 324-328.

Nasevicius A, Ekker SC. 2000. Effective targeted gene 'knockdown' in zebrafish. Nat Genet 26: 216-220.

Oliver TG, Grasfeder LL, Carroll AL, Kaiser C, Gillingham CL, Lin SM, Wickramasinghe R, Scott MP, Wechsler-Reya RJ. 2003. Transcriptional profiling of the Sonic hedgehog response: A critical role for N-myc in proliferation of neuronal precursors. Proc Natl Acad Sci 100: 7331-7336.

Parra LM, Zou Y. 2010. Sonic hedgehog induces response of commissural axons to Semaphorin repulsion during midline crossing. Nat Neurosci 13: 29-35.

Prahst C, Heroult M, Lanahan AA, Uziel N, Kessler O, ShragaHeled N, Simons M, Neufeld G, Augustin HG. 2008. Neuropilin-1-VEGFR-2 complexing requires the PDZ-binding domain of neuropilin-1. I Biol Chem 283: 25110-25114.

Resende TP, Ferreira M, Teillet MA, Tavares AT, Andrade RP, Palmeirim I. 2010. Sonic hedgehog in temporal control of somite formation. Proc Natl Acad Sci 107: 12907-12912.
Ribes V, Briscoe J. 2009. Establishing and interpreting graded Sonic Hedgehog signaling during vertebrate neural tube patterning: The role of negative feedback. Cold Spring Harb Perspect Biol 1: a002014. doi: 10.1101/cshperspect.a002014.

Rohatgi R, Scott MP. 2007. Patching the gaps in Hedgehog signalling. Nat Cell Biol 9: 1005-1009.

Rohatgi R, Milenkovic L, Scott MP. 2007. Patched1 regulates hedgehog signaling at the primary cilium. Science 317: 372 376.

Rohatgi R, Milenkovic L, Corcoran RB, Scott MP. 2009. Hedgehog signal transduction by Smoothened: Pharmacologic evidence for a 2-step activation process. Proc Natl Acad Sci 106: 3196-3201.

Rossignol M, Gagnon ML, Klagsbrun M. 2000. Genomic organization of human neuropilin-1 and neuropilin-2 genes: Identification and distribution of splice variants and soluble isoforms. Genomics 70: 211-222.

Shimizu M, Murakami Y, Suto F, Fujisawa H. 2000. Determination of cell adhesion sites of neuropilin-1. J Cell Biol 148: 1283-1293.

Soker S, Takashima S, Miao HQ, Neufeld G, Klagsbrun M. 1998. Neuropilin-1 is expressed by endothelial and tumor cells as an isoform-specific receptor for vascular endothelial growth factor. Cell 92: 735-745.

Soker S, Miao HQ, Nomi M, Takashima S, Klagsbrun M. 2002. VEGF165 mediates formation of complexes containing VEGFR-2 and neuropilin-1 that enhance VEGF165-receptor binding. J Cell Biochem 85: 357-368.

St-Jacques B, Dassule HR, Karavanova I, Botchkarev VA, Li J, Danielian PS, McMahon JA, Lewis PM, Paus R, McMahon AP. 1998. Sonic hedgehog signaling is essential for hair development. Curr Biol 8: 1058-1068.

Suto F, Ito K, Uemura M, Shimizu M, Shinkawa Y, Sanbo M, Shinoda T, Tsuboi M, Takashima S, Yagi T, et al. 2005. Plexin-a4 mediates axon-repulsive activities of both secreted and transmembrane semaphorins and plays roles in nerve fiber guidance. J Neurosci 25: 3628-3637.

Taipale J, Chen JK, Cooper MK, Wang B, Mann RK, Milenkovic L, Scott MP, Beachy PA. 2000. Effects of oncogenic mutations in Smoothened and Patched can be reversed by cyclopamine. Nature 406: 1005-1009.

Takagi S, Kasuya Y, Shimizu M, Matsuura T, Tsuboi M, Kawakami A, Fujisawa H. 1995. Expression of a cell adhesion molecule, neuropilin, in the developing chick nervous system. Dev Biol 170: 207-222.

Takashima S, Kitakaze M, Asakura M, Asanuma H, Sanada S, Tashiro F, Niwa H, Miyazaki Ji J, Hirota S, Kitamura Y, et al. 2002. Targeting of both mouse neuropilin-1 and neuropilin-2 genes severely impairs developmental yolk sac and embryonic angiogenesis. Proc Natl Acad Sci 99: 3657-3662.

Thisse C, Thisse B. 2008. High-resolution in situ hybridization to whole-mount zebrafish embryos. Nat Protoc 3: 59-69.

Valdembri D, Caswell PT, Anderson KI, Schwarz JP, Konig I, Astanina E, Caccavari F, Norman JC, Humphries MJ, Bussolino F, et al. 2009. Neuropilin-1/GIPC1 signaling regulates $\alpha 5 \beta 1$ integrin traffic and function in endothelial cells. PLOS Biol 7: e25. doi: 10.1371/journal.pbio.1000025.

van Amerongen R, Nusse R. 2009. Towards an integrated view of Wnt signaling in development. Development 136: 3205-3214.

van Eeden FJ, Granato M, Schach U, Brand M, Furutani-Seiki M, Haffter P, Hammerschmidt M, Heisenberg CP, Jiang YJ, Kane DA, et al. 1996. Mutations affecting somite formation and patterning in the zebrafish, Danio rerio. Development 123: 153-164.

Ventura A, Meissner A, Dillon CP, McManus M, Sharp PA, Van Parijs L, Jaenisch R, Jacks T. 2004. Cre-lox-regulated condi- 
Hillman et al.

tional RNA interference from transgenes. Proc Natl Acad Sci 101: 10380-10385.

Wang B, Fallon JF, Beachy PA. 2000. Hedgehog-regulated processing of Gli3 produces an anterior/posterior repressor gradient in the developing vertebrate limb. Cell 100: 423-434.

Wen X, Lai CK, Evangelista M, Hongo JA, de Sauvage FJ, Scales SJ. 2010. Kinetics of hedgehog-dependent full-length Gli3 accumulation in primary cilia and subsequent degradation. Mol Cell Biol 30: 1910-1922.

Yaron A, Huang PH, Cheng HJ, Tessier-Lavigne M. 2005. Differential requirement for Plexin-A3 and -A4 in mediating responses of sensory and sympathetic neurons to distinct class 3 Semaphorins. Neuron 45: 513-523.

Zou Y, Stoeckli E, Chen H, Tessier-Lavigne M. 2000. Squeezing axons out of the gray matter: A role for slit and semaphorin proteins from midline and ventral spinal cord. Cell 102: 363-375. 


\section{Erratum}

Genes \& Development 25: 2333-2346 (2011)

Neuropilins are positive regulators of Hedgehog signal transduction

R. Tyler Hillman, Brian Y. Feng, Jun Ni, Wei-Meng Woo, Ljiljana Milenkovic, Melanie G. Hayden Gephart,

Mary N. Teruel, Anthony E. Oro, James K. Chen, and Matthew P. Scott

In the above-mentioned article, the following funding should have been mentioned in the Acknowledgments section:

J.K.C. also gratefully acknowledges support from an American Cancer Society Research Scholar Grant (RSG-08-041-01$\mathrm{DDC})$.

The authors apologize for the omission. 


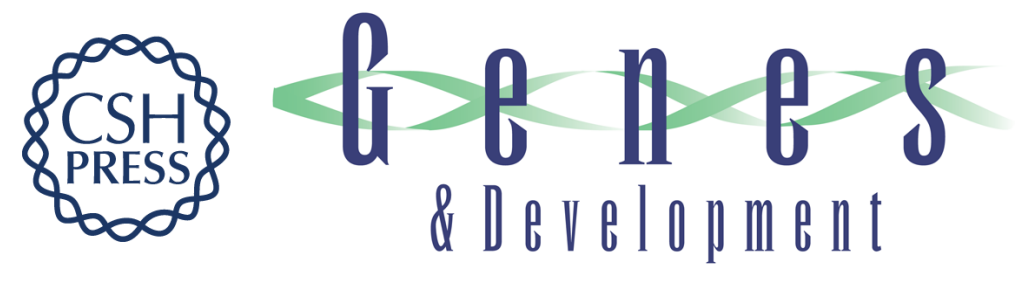

\section{Neuropilins are positive regulators of Hedgehog signal transduction}

R. Tyler Hillman, Brian Y. Feng, Jun Ni, et al.

Genes Dev. 2011, 25: originally published online November 3, 2011

Access the most recent version at doi:10.1101/gad.173054.111

\section{Supplemental http://genesdev.cshlp.org/content/suppl/2011/10/26/gad.173054.111.DC1 \\ Material}

Related Content Neuropilins are positive regulators of Hedgehog signal transduction

R. Tyler Hillman, Brian Y. Feng, Jun Ni, et al.

Genes Dev. March , 2012 26: 630 New Nrps Nexus

Annalisa M. VanHook

Sci. Signal. November , 2011 4: ec325

References This article cites 73 articles, 32 of which can be accessed free at:

http://genesdev.cshlp.org/content/25/22/2333.full.html\#ref-list-1

Articles cited in:

http://genesdev.cshlp.org/content/25/22/2333.full.html\#related-urls

License Freely available online through the Genes \& Development Open Access option.

Email Alerting
Service $\begin{aligned} & \text { Receive free email alerts when new articles cite this article - sign up in the box at the top } \\ & \text { right corner of the article or click here. }\end{aligned}$

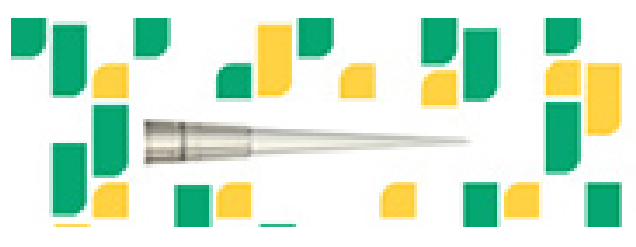

Focused on your science. 